\title{
Realistic Models for the Comet Dynamical Environment
}

\author{
Sharyl M. Byram* and Daniel J. Scheeres ${ }^{\dagger}$ \\ Department of Aerospace Engineering, University of Michigan, Ann Arbor, MI 48109 \\ Michael R. Combi $\ddagger$ \\ Department of Atmospheric, Oceanic, and Space Sciences, University of Michigan, Ann Arbor, MI 48109
}

\begin{abstract}
An outgassing jet model is presented in support of spacecraft navigation for future missions to comets. The outgassing jet is modelled as an emission cone while the comet is modelled as a uniform density triaxial ellipsoid. The comet's motion about the Sun is included in the model. This model is used for simulation and estimation of the physical outgassing properties of jets at and near the surface of a comet.
\end{abstract}

\section{Nomenclature}

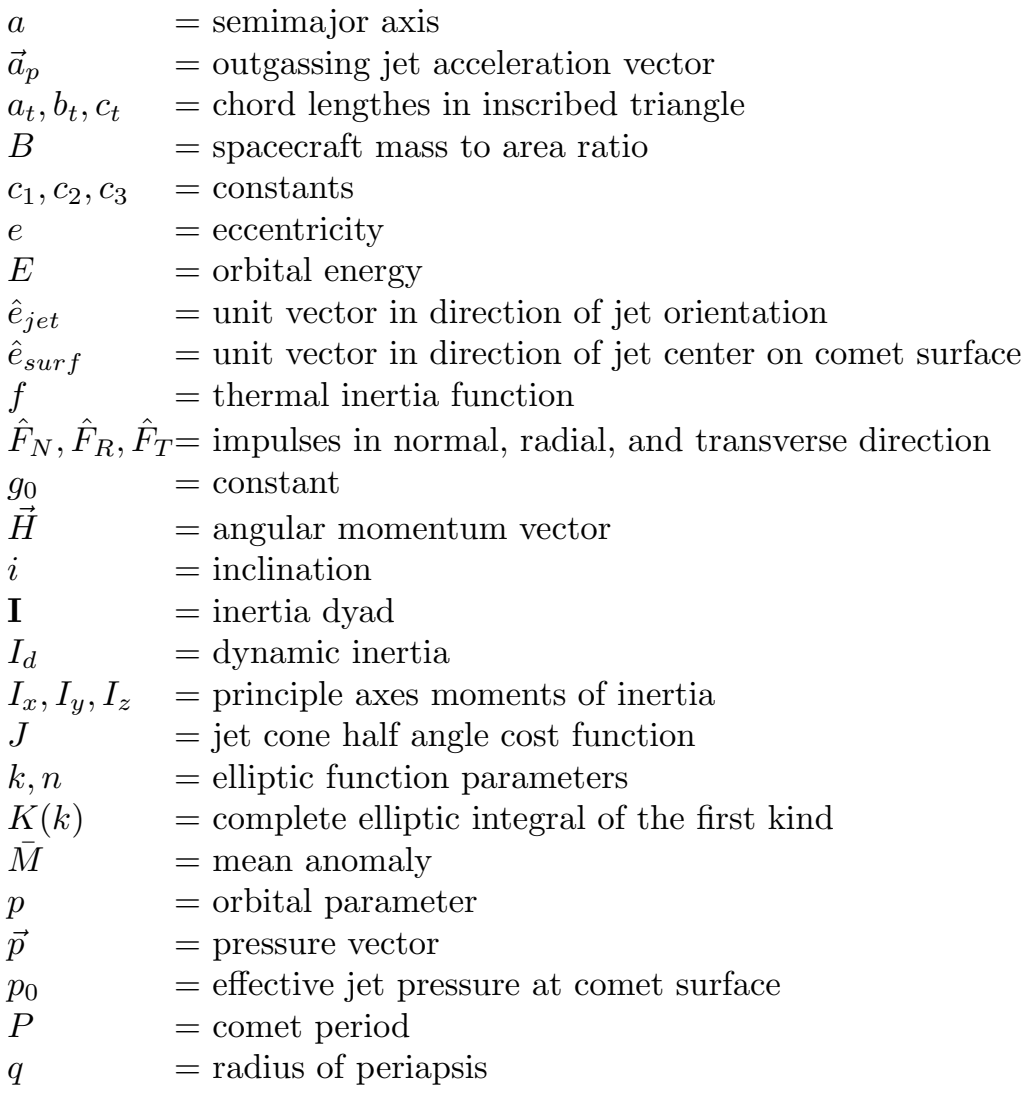

\footnotetext{
${ }^{*}$ Graduate Student, Department of Aerospace Engineering, University of Michigan, Ann Arbor, MI 48109, byramsm@umich.edu, Student Member.

${ }^{\dagger}$ Associate Professor, Department of Aerospace Engineering, University of Michigan, Ann Arbor, MI 48109, scheeres@umich.edu, Professional Member.

${ }^{\ddagger}$ Research Professor, Department of Atmospheric, Oceanic, and Space Sciences, University of Michigan, Ann Arbor, MI 48109, mcombi@umich.edu.
} 


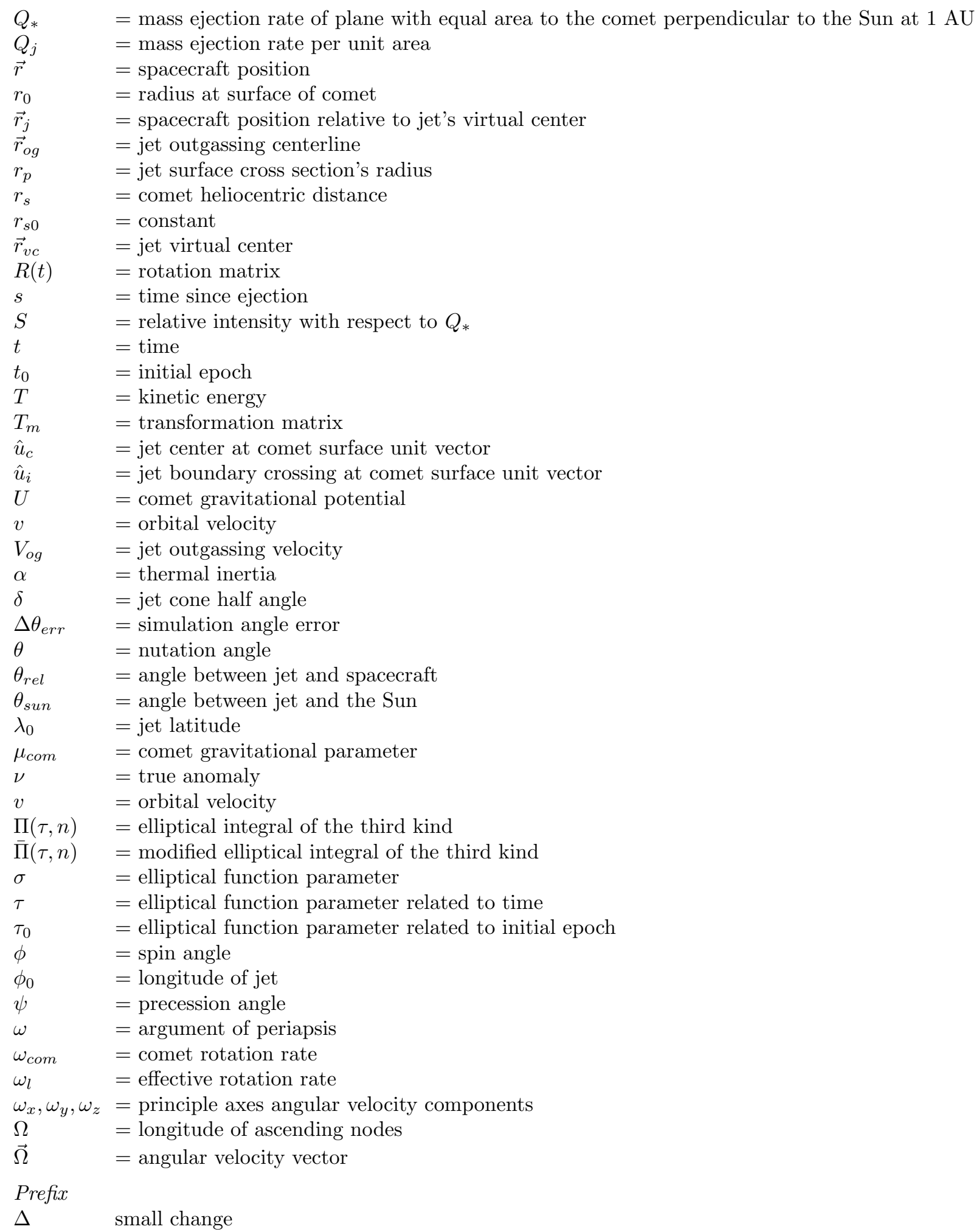

\section{Introduction}

With the return of the Stardust capsule back to Earth in January, the interest in cometary science has grown. The space agencies around the world have sent an increasing number of missions to comets in our solar system as well as to asteroids. Most recently, NASA launched Stardust in 1999 which reached the comet

$$
2 \text { of } 16
$$


P/Wild 2 in January 2004 and returned a sample of dust and volatiles from the coma of the comet. In July 2005, NASA's Deep Impact mission provided to the world high-resolution images of the surface of the comet Tempel 1 as well as impacting the surface to gain knowledge of its physical properties. ESA launched the Rosetta mission in 2004 and is scheduled to reach comet $67 \mathrm{P} /$ Churyumov-Gerasimenko in 2014 and deliver a lander to the surface of the comet. These missions can provide an abundance of scientific information about the physical properties of comets, but they are also of interest in the field of orbital dynamics.

Orbit determination of a spacecraft in orbit about a comet has been explored previously. Miller, et. al. ${ }^{1}$ modelled the outgassing of a comet as constant bias non-gravitational acceleration with a variable modelling error acceleration from an active region which is defined by a portion of the surface area of an ellipsoid. Scheeres, et. al. ${ }^{2}$ approached the outgassing acceleration in two cases to determine the stability of the spacecraft in terms of crashing into the comet or escaping from the comet. Their first outgassing model assumes that the outgassing field is continuous but variable depending on the angle made with the Sun. Their second model assumes that the comet has jets emanating from the surface, with spacecraft interaction only acting in the vicinity of the jets.

Due to the similarity in shape and size, comets yield a set of dynamical problems common to that of asteroids, but it is the outgassing fields that make the dynamical environment of comets unique. It is the description of the outgassing fields and their dynamical implications that will be the focus of this paper. There exist many theories about the structure of a comet's outgassing fields. Crifo, et $\mathrm{al}^{3}$ theorize that the coma structure is produced by multiple interacting dusty gas jets. Sekanina, et al ${ }^{4}$ use a model of comet 81P/Wild 2 from images taken by Stardust of dust particles, which are ejected from a small active source, which make up a thin conical sheet in the coma before the formation is gradually dispersed by various forces. They discuss 20 jets imaged on comet $81 \mathrm{P} /$ Wild2 oriented in an arbitrary direction from the surface. For the purpose of this research, it will be assumed that the outgassing pressure field is created by a collection of discrete jets on the surface of the comet similar to the ones described in Sekanina, et al. ${ }^{4}$ Our model, unlike others, will have a full three dimensional geometry. This paper addresses the need for a spacecraft to navigate these discrete jet outgassing fields while in orbit about a comet. We will define and explore a simple model for an outgassing jet from a surface of a comet while considering its implications. In addition to the model and simulation, the paper discusses how the parameters of this model can be estimated using navigational data from a spacecraft's passages through the jet's outgassing field and uses the jets identified by Sekanina, et $\mathrm{al}^{4}$ to simulate a spacecraft's trajectory about an idealized comet 81/P Wild2.

\section{Equations of Motion}

To begin, it is assumed that the comet is located significantly far from any other major celestial body such that the spacecraft's motion about the comet follows the two-body orbital equations. The mass of the spacecraft is assumed to be significantly smaller than that of the comet, such that the equations of motion are of the form:

$$
\ddot{\vec{r}}=\frac{\partial U}{\partial \vec{r}}+\vec{a}_{p}
$$

where $\vec{r}$ is the spacecraft's inertial position vector relative to the comet's center of mass, $U$ is the comet's gravitational potential, and $\vec{a}_{p}$ is the outgassing jet acceleration applied to the spacecraft. We assume that the jet is oriented in some arbitrary direction relative to the comet fixed frame and will diminish as $1 /\left|\vec{r}-\vec{r}_{v c}\right|^{2}$, where $\vec{r}_{j}=\vec{r}-\vec{r}_{v c}$ is the spacecraft's position vector relative to the virtual center of the jet. This assumption leads to the basic equation for the outgassing acceleration:

$$
\vec{a}_{p}=\frac{p_{0}\left(\overrightarrow{r_{j}}\right)}{B} \frac{\overrightarrow{r_{j}}}{\left|\overrightarrow{r_{j}}\right|^{3}}
$$

where $B=m / A$ is the spacecraft's mass to area ratio and $p_{0}\left(\overrightarrow{r_{j}}\right)$ is the effective pressure field at the comet surface. Equation 2 provides the basis of the jet outgassing model.

\section{Outgassing Jet Model}

This paper defines and explores an emission cone model for a outgassing jet from the surface of a comet. It also gives a detailed description based on Stardust's data of the Wild 2 comet found in Sekanina, ${ }^{4}$ as well 
as a general description of this model and its implications. It explores the estimation of the parameters of this model using navigation data. First presented is the basic definition and features of the model.

\section{A. Comet Assumptions}

Previous work has been done with a comet model that is a perfectly spherical body with constant rotation, $\omega_{\text {com }}$, about the $z$-axis in an inertial frame. Since this is rarely the case, this paper will address the physical properties of the comet in a more realistic model. The comet will be assumed be an ellipsoid with principle half lengths which represents the best fit to images taken in respect to size and shape. Scheeres ${ }^{5}$ describes the gravitational field for an ellipsoid. The mass distribution of this comet model is assumed, for now, to have uniform mass density. This, however, is only an approximation to the true comet body. The rotational properties of the comet body are assumed to be constant rotation, $\omega_{\text {com }}$, about the $z$-axis in an body fixed frame which may be inclined to the comet's orbital plane. For an ellipsoid with equal principle half lengths, the model collapses to the special case of a spherical shape which yields a point mass gravity field .

\section{B. Outgassing Jet}

In our simplest model, the outgassing field of the comet is assumed to be produced by a single discrete jet located on the surface of the comet, although the simulated model includes multiple jets of varying strength. The jet is assumed to be fixed on the comet surface and its center is located at a radius of $r_{0}$, longitude of $\phi_{0}$, and latitude of $\lambda_{0}$ in the body fixed frame.

The active region on the surface of the comet is assumed to have a circular cross section where the size and shape of the jet is defined by a constant half angle, $\delta$, and surface radius, $r_{p}$. These two parameters along with the jet orientation define the location of the virtual center of the jet, $\vec{r}_{j}$. It is assumed that the gas travels at a constant velocity, $V_{o g}$, in a direction defined by the jet's orientation which may point in any arbitrary direction away from the comet surface. We note that this assumption may not hold close to the comet surface where complex gas dynamics and interactions are occurring. ${ }^{6}$ The centerline of an outgassing jet becomes a function of time since ejection, $s$, and can be expressed in the comet body fixed frame (illustrated in Fig. 1):

$$
\vec{r}_{o g}(s)=r_{0} \hat{e}_{r_{\text {surf }}}+V_{\text {og }} s \hat{e}_{r_{j e t}}
$$

where $\hat{e}_{r_{\text {surf }}}$ is the body fixed unit vector pointing from

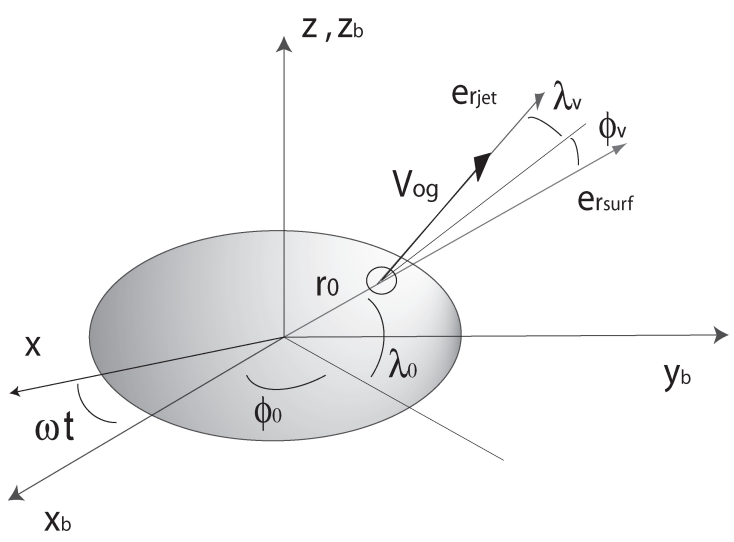

Figure 1. Coordinate frames the center of the comet to the jet surface point in the radial direction and $\hat{e}_{r_{j e t}}$ is the body fixed unit vector pointing in the jet orientation direction. This frame rotates with the comet at a constant rate, $\omega_{\text {com }}$, and is transformed from a non-rotating coordinate system with $z$-axis aligned along the spin axis of the comet, to the body fixed rotating coordinate frame by:

$$
R(t)=\left[\begin{array}{ccc}
\cos \left(\lambda_{0}\right) \cos \left(\phi_{0}+\omega_{\text {com }} t\right) & \cos \left(\lambda_{0}\right) \sin \left(\phi_{0}+\omega_{\text {com }} t\right) & \sin \left(\lambda_{0}\right) \\
-\sin \left(\phi_{0}+\omega_{\text {com }} t\right) & \cos \left(\phi_{0}+\omega_{\text {com }} t\right) & 0 \\
-\sin \left(\lambda_{0}\right) \cos \left(\phi_{0}+\omega_{\text {com }} t\right) & -\sin \left(\lambda_{0}\right) \sin \left(\phi_{0}+\omega_{\text {com }} t\right) & \cos \left(\lambda_{0}\right)
\end{array}\right]
$$

The jet outgassing centerline is then expressed as a function of both the time, $t$, as well as the time since ejection, $s$.

$$
\vec{r}_{o g}(t, s)=R(t)^{T}\left[r_{0} \hat{e}_{r_{s u r f}}+V_{o g} s \hat{e}_{r_{j e t}}\right]
$$

Since a circular cross section is assumed, the surface of the outgassing jet is modelled as a solid cone that can be defined by the constant half angle, $\delta$, from the centerline as well as the time since ejection, $s$, in the body fixed frame. This outgassing jet will generate a pressure field which is a function of the mass ejection rate per unit area, $Q_{j}$, of the jet at the surface of the comet and the velocity of the material being ejected, $V_{o g}$. Therefore the pressure of the outgassing at the surface of the comet becomes:

$$
p_{0}=Q_{j} V_{o g}
$$

$$
4 \text { of } 16
$$


As the velocity field is assumed to be uniformly outwards, we define a vector pressure aligned with the velocity field in the direction of the jet's orientation as:

$$
\vec{p}_{0}=Q_{j} V_{o g} \hat{e}_{r_{j e t}}
$$

The mass ejection rate of the jet can be estimated as: ${ }^{7}$

$$
Q_{j}=S f\left(\theta_{\text {sun }}\right) g\left(r_{s}\right) Q_{*}
$$

where $Q_{*}$ is the mass ejection rate of a plane with an area equal to the surface area of the comet perpendicular to the Sun at a distance of 1 AU away, $S$ is the relative intensity with respect to $Q_{*}, \theta_{\text {sun }}$ is the angle between the vector pointing in the direction of the Sun and the normal vector of the outgassing jet, and $r_{s}$ is the distance from the comet to the Sun. As the comet travels closer to the Sun its thermal activity will increase as a function of $r_{s}$. The outgassing strength has been empirically determined to follow the law:

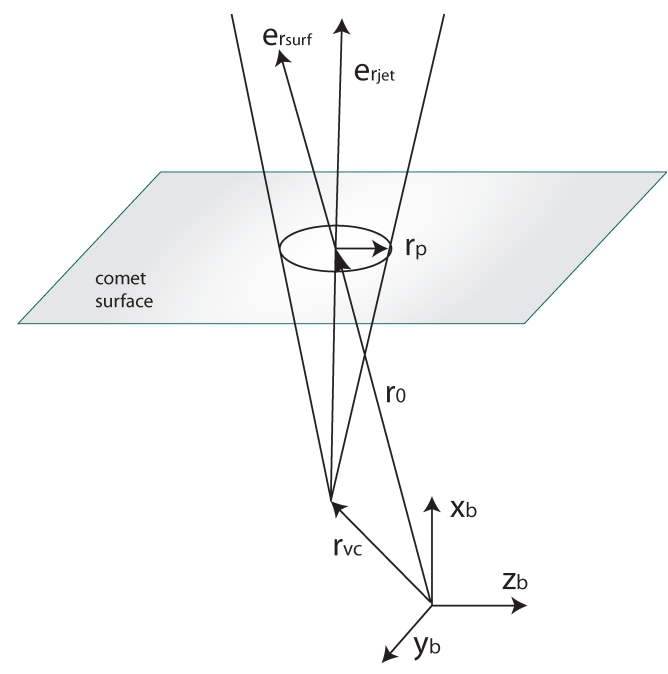

Figure 2. Outgassing Jet Surface

$$
g\left(r_{s}\right)=g_{0}\left(\frac{r_{s}}{r_{s 0}}\right)^{-c_{1}}\left[1+\left(\frac{r_{s}}{r_{s 0}}\right)^{c_{2}}\right]^{-c_{3}}
$$

where $c_{1}=2.15, c_{2}=5.093, c_{3}=4.6142, r_{s 0}=2.808$, and $g_{0}=0.111262$ from Marsden, et al. ${ }^{8}$ The strength of the pressure at the surface of the comet is related to the angle the Sun makes with the normal to the jet, given by $f\left(\theta_{\text {sun }}\right)$. If the unit vector towards the Sun is defined as $\hat{u}_{s}$, then $\cos \theta_{\text {sun }}=\hat{u}_{s} \cdot \hat{r}_{\text {og }}(t, s)$. This function provides for a strong pressure when the surface is illuminated by the Sun and a weak (possibly zero) pressure when it is not.

$$
f\left(\theta_{\text {sun }}\right)=\max \left\{\begin{array}{c}
0 \\
1-\alpha\left(1-\cos \theta_{\text {sun }}\right)
\end{array}\right.
$$

where the parameter $\alpha$ is related to the thermal inertia, and can have any value such that $0 \leq \alpha \leq 1$. The pressure magnitude felt by the orbiter is assumed to be inversely proportional to the radial distance from the virtual center of the jet such that the pressure vector at the orbiter's position becomes:

$$
\vec{p}=\vec{p}_{0}\left(\frac{r_{0}}{\left|\overrightarrow{r_{j}}\right|}\right)^{2}
$$

where $\left|\overrightarrow{r_{j}}\right|$ is the distance from the virtual center of the jet to the orbiter and $\left|\overrightarrow{r_{j}}\right|>r_{0}$ is assumed. Then the acceleration felt by the spacecraft from the outgassing pressure is of the form:

$$
\vec{a}_{p}=\frac{\vec{p}}{B}=\vec{p}_{0} \frac{1}{B}\left(\frac{r_{0}}{\left|\overrightarrow{r_{j}}\right|}\right)^{2}
$$

where $B$ is the ratio of the mass of the spacecraft to the area of the spacecraft normal to the comet.

\section{Comet 81/P Wild 2 Model Assumptions}

The model as described is dependent on real values for the outgassing jet velocity and half angle as well as the size, shape, and mass of the comet for a simulation. Through analysis of images taken of comet $81 / \mathrm{P}$ Wild 2, locations and orientations of 20 jets have been made by Sekanina, et $\mathrm{l}^{4}$ and can be found in the Appendix. The size, shape, and mass have also been estimated. The simulated model will use the principle half lengths defined by JPL of $2750 \mathrm{~km}, 2000 \mathrm{~km}$, and $1650 \mathrm{~km}$. General outgassing velocities been estimated to range from $350 \mathrm{~m} / \mathrm{s}$ by Crifo, et al ${ }^{3}$ to $900 \mathrm{~m} / \mathrm{s}$ by Combi, et al. ${ }^{6}$ In general, the jet half angle has received less attention but analysis of Hale-Bopp has indicated discrete jets with estimated half angles of up to 10 degrees $^{6}$ and spherical squares with side lengths up to 50 degrees have been used by Crifo, et $\mathrm{al}^{3}$ to describe larger jets.

$$
5 \text { of } 16
$$




\section{Orbit Mechanics about an Outgassing Jet}

\section{A. Orbital Elements}

In order to predict what will happen when a spacecraft flies through a jet outgassing field, we start with the analysis of small changes in orbital elements. First, we note that for large distances from the comet, the radial component of the outgassing is dominant and therefore the outgassing due to a jet passage will be considered to be a radial impulse. We begin with the orbital parameter, $p$ :

$$
p=a\left(1-e^{2}\right)
$$

First we note that $p$ will be conserved ${ }^{2}$ through a jet passage as it is related to the angular momentum which does not change under a radial impulse. Taking a small change in $p, \Delta p$, yields the following:

$$
\Delta p=\Delta a\left(1-e^{2}\right)-2 a e \Delta e
$$

The same thing is done for the orbital energy to find a small change in semimajor axis, $a$.

$$
\begin{gathered}
E=-\frac{\mu_{\text {com }}}{2 a} \\
\Delta E=\frac{\mu_{\text {com }} \Delta a}{2 a^{2}} \\
\Delta a=\frac{2 a^{2} \Delta E}{\mu_{\text {com }}}
\end{gathered}
$$

It is seen in Eq. 17 that a positive change in energy increases the semimajor axis and that a negative change in energy results in a decrease in the semimajor axis. Substituting this result into Eq. 14 and knowing that $p$ is conserved $(\Delta p=0)$, yields that a positive change in energy increases the eccentricity and that a negative change in energy results in a decrease in the eccentricity.

$$
\begin{gathered}
0=\frac{2 a^{2} \Delta E}{\mu_{c o m}}\left(1-e^{2}\right)-2 a e \Delta e \\
\Delta e=\frac{a \Delta E}{\mu_{c o m} e}\left(1-e^{2}\right)
\end{gathered}
$$

In terms of a jet passage, the energy equation as a function of velocity provides additional insight.

$$
E=\frac{1}{2} v^{2}-\frac{\mu_{\text {com }}}{|\vec{r}|}
$$

where $v$ is the spacecraft's velocity magnitude. Variations in energy will arise due to impulsive changes in the spacecraft's velocity:

$$
\Delta E=v \Delta v
$$

Substituting this $\Delta E$ result in Eqs. 17] and 19, yields:

$$
\begin{gathered}
\Delta a=\frac{2 a^{2} v \Delta v}{\mu_{\text {com }}} \\
\Delta e=\frac{a v \Delta v}{\mu_{\text {com }} e}\left(1-e^{2}\right)
\end{gathered}
$$

From Eqs. 22 and 23 it can be seen that a positive change in velocity increases the semimajor axis and eccentricity and that a negative change in velocity results in a decrease in the semimajor axis and eccentricity. Since the outgassing primarily acts in the radial direction, the radial component of the spacecraft's velocity is the only effected component. Therefore, if the spacecraft has a negative radial velocity component (Fig. 3 ) such as when it is travelling from apoapsis to periapsis, the outgassing jet will tend to circularize the orbit, or decrease the semimajor axis and eccentricity. Likewise, if the spacecraft has a positive radial velocity component such as when it is travelling from periapsis to apoapsis, the outgassing jet will tend to make the orbit more eccentric and increase the semimajor axis.

$$
6 \text { of } 16
$$


The radius of periapsis, $q$, is also of interest. Taking a small change in the radius of periapsis yields:

$$
\begin{gathered}
q=a(1-e) \\
\Delta q=\Delta a(1-e)-a \Delta e
\end{gathered}
$$

This can be related to a change in velocity by substituting in Eqs. 22 and 23 .

$$
\Delta q=\frac{-q^{2} V \Delta V}{\mu_{c o m} e}
$$

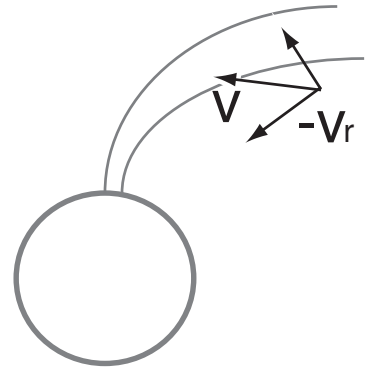

(a)

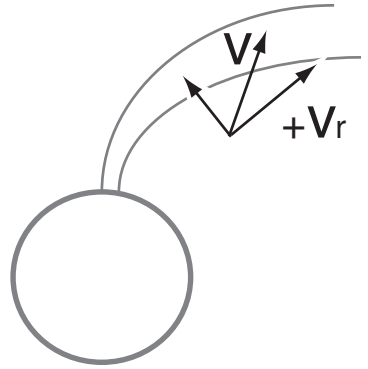

(b)

This results in an increase in radius of periapsis when the spacecraft has a negative radial velocity

Figure 3. Spacecraft Velocity Components: (a) Negative Radial Component, (b) Positive Radial Component component during a jet passage. Likewise, a decrease in radius of periapsis occurs when the spacecraft has a positive radial velocity component during a jet passage.

\section{B. Lagrange Planetary Equations}

Changes in the full set of orbital elements can be explored by considering at the Lagrange planetary equations. Starting with these equations in the Gaussian form:

$$
\begin{aligned}
\frac{d a}{d t}= & \frac{2 a^{2} e}{h} \sin \nu \hat{F}_{R}+\frac{2 a^{2} h}{\mu_{c o m} r} \hat{F}_{T} \\
\frac{d e}{d t}= & \frac{h}{\mu_{\operatorname{com}}}\left(\sin \nu \hat{F}_{R}+\frac{e+2 \cos \nu+e \cos ^{2} \nu}{1+e \cos / n u} \hat{F}_{T}\right) \\
\frac{d i}{d t}= & \frac{r}{h} \cos \varpi \hat{F}_{N} \\
\frac{d \Omega}{d t}= & \frac{r \sin \varpi}{h \sin i} \hat{F}_{N} \\
\frac{d \omega}{d t}= & -\frac{h}{\mu_{c o m} e} \cos \nu \hat{F}_{R}-\frac{r}{h} \cot i \sin \varpi \hat{F}_{N}+\frac{\left(h^{2}+r \mu_{c o m}\right) \sin \nu}{\mu_{c o m} e h} \hat{F}_{T} \\
\frac{d \bar{M}}{d t}= & n-\frac{1}{n a}\left(\frac{2 r}{a}-\frac{\left(1-e^{2}\right)}{e} \cos \nu\right) \hat{F}_{R} \\
& -\frac{\left(1-e^{2}\right)}{n a e}\left(1+\frac{r}{p}\right) \sin \nu \hat{F}_{T}
\end{aligned}
$$

where

$$
\begin{gathered}
h=n a^{2} \sqrt{1-e^{2}} \\
n=\sqrt{\frac{\mu_{c o m}}{a^{3}}} \\
\varpi=\omega+\nu \\
r=|\vec{r}|
\end{gathered}
$$

and $\hat{F}_{R}, \hat{F}_{T}$, and $\hat{F}_{N}$ are accelerations in the radial, transverse, and orbit normal directions, respectively, and $\nu$ is the true anomaly. For jet passages, we have assumed that the outgassing component in the radial direction is dominant, therefore the acceleration terms in Eqs. 27 to 32 become:

$$
\begin{gathered}
\hat{F}_{R}=a_{p}=p_{0} \frac{1}{B}\left(\frac{r_{0}}{\left|\vec{r}_{j}\right|}\right)^{2} \\
\hat{F}_{T}=0 \\
\hat{F}_{N}=0
\end{gathered}
$$

$$
7 \text { of } 16
$$


where $r_{0}$ is the comet radius at the jet location, and $\vec{r}_{j}$ is the position of the spacecraft relative to the virtual center of the jet. The Lagrange planetary equations for the outgassing acceleration become:

$$
\begin{aligned}
\frac{d a}{d t} & =\frac{2 a^{2} e}{h} \sin \nu p_{0} \frac{1}{B}\left(\frac{r_{0}}{\left|\vec{r}_{j}\right|}\right)^{2} \\
\frac{d e}{d t} & =\frac{h}{\mu_{c o m}} \sin \nu p_{0} \frac{1}{B}\left(\frac{r_{0}}{\left|\vec{r}_{j}\right|}\right)^{2} \\
\frac{d i}{d t} & =0 \\
\frac{d \Omega}{d t} & =0 \\
\frac{d \omega}{d t} & =-\frac{h}{\mu_{c o m} e} \cos \nu p_{0} \frac{1}{B}\left(\frac{r_{0}}{\left|\vec{r}_{j}\right|}\right)^{2} \\
\frac{d \bar{M}}{d t} & =n-\frac{1}{n a}\left(\frac{2 r}{a}-\frac{\left(1-e^{2}\right)}{e} \cos \nu\right) p_{0} \frac{1}{B}\left(\frac{r_{0}}{\left|\vec{r}_{j}\right|}\right)^{2}
\end{aligned}
$$

We note that the radial acceleration will always be positive since the outgassing pressure acts only in the outward radial direction. It can be seen that the outgassing pressure affects four of the elements: the semimajor axis, $a$, the eccentricity, $e$, the argument of perigee, $\omega$, and the mean anomaly, $\bar{M}$. The $\sin \nu$ term will be positive while the spacecraft is located along the periapsis to apoapsis portion of its orbit where it has a positive radial component of its velocity. Likewise, the $\sin \nu$ term will be negative while the spacecraft is located along the apoapsis to periapsis portion of its orbit where it has a negative radial component of its velocity. As seen in semimajor axis and eccentricity equations in Eqs. 40 and 41, the semimajor axis and the eccentricity will increase while the spacecraft's radial velocity is positive and will decrease while the spacecraft's radial velocity is negative as a result of the $\sin \nu$ term. As noted previously, the parameter $p$ is conserved. This can be proven using the results of a radial acceleration in the Lagrange planetary equations.

$$
\begin{gathered}
\frac{d p}{d t}=\frac{d a}{d t}\left(1-e^{2}\right)-2 a e \frac{d e}{d t} \\
\frac{d p}{d t}=\frac{2 a^{2} e}{h} \sin \nu p_{0} \frac{1}{B}\left(\frac{r_{0}}{\left|\vec{r}_{j}\right|}\right)^{2}\left(1-e^{2}\right)-2 a e \frac{h}{\mu_{c o m}} \sin \nu p_{0} \frac{1}{B}\left(\frac{r_{0}}{\left|\vec{r}_{j}\right|}\right)^{2}=0
\end{gathered}
$$

From there, we can get estimates for changes in orbit due to a jet passage. If we assume that the comet spins faster than the orbit travels, the time it takes for the jet to sweep over a spacecraft located at the jet longitude is approximately:

$$
\Delta t=\frac{2 \delta}{\omega_{c o m} \cos \lambda_{0}}
$$

This leads to impulsive changes in orbital elements of

$$
\begin{aligned}
\Delta a & =\frac{d a}{d t} \Delta t=\frac{2 a^{2} e}{h} \sin \nu p_{0} \frac{1}{B}\left(\frac{r_{0}}{\left|\vec{r}_{j}\right|}\right)^{2} \frac{2 \delta}{\omega_{c o m} \cos \lambda_{0}} \\
\Delta e & =\frac{d e}{d t} \Delta t=\frac{h}{\mu_{c o m}} \sin \nu p_{0} \frac{1}{B}\left(\frac{r_{0}}{\left|\vec{r}_{j}\right|}\right)^{2} \frac{2 \delta}{\omega_{c o m} \cos \lambda_{0}} \\
\Delta \omega & =\frac{d \omega}{d t} \Delta t=-\frac{h}{\mu_{\text {com }} e} \cos \nu p_{0} \frac{1}{B}\left(\frac{r_{0}}{\left|\vec{r}_{j}\right|}\right)^{2} \frac{2 \delta}{\omega_{c o m} \cos \lambda_{0}}
\end{aligned}
$$

Note that if the spacecraft travels opposite to the comet's rotation, the impulse will be less if the spacecraft is travels in the same sense as the comet's rotation.

\section{Simulation}

A simulation was developed incorporating the above model. The spacecraft was assumed to follow the trajectory described by solving the standard two-body problem while the spacecraft is not within an outgassing

$$
8 \text { of } 16
$$


region. The pressure acceleration, $\vec{a}_{p}$, is applied to the spacecraft when it is determined that the angle between the outgassing pressure vector at the same radius as the spacecraft and the spacecraft position vector, $\theta_{\text {rel }}$, is within a defined angle error, $\Delta \theta_{\text {err }}$, of the half angle defining the active region's size. The angle $\theta_{r e l}$ is determined by:

$$
\theta_{r e l}=\arccos \frac{\vec{r}_{o g} \cdot \vec{r}}{\left\|\vec{r}_{o g}\right\|\|\vec{r}\|}
$$

where $\vec{r}$ is the position vector of the spacecraft given in the same coordinate frame as $\vec{r}_{o g}$.

Table 1. Simulation Parameters

\begin{tabular}{llcl} 
Variable & Description & Value & Unit \\
\hline$x$ & comet principle length & 2.75 & $\mathrm{~km}$ \\
$y$ & comet principle length & 2.00 & $\mathrm{~km}$ \\
$z$ & comet principle length & 1.65 & $\mathrm{~km}$ \\
$P$ & comet period & 12 & $\mathrm{hr}$ \\
$V_{o g}$ & outgassing radial velocity & 0.5 & $\mathrm{~km} / \mathrm{s}$ \\
$\omega_{\text {com }}$ & comet rotation rate & $\frac{2 \pi}{T}$ & $\mathrm{rad} / \mathrm{s}$ \\
$\delta$ & jet shape half angle & 1.5 & $\mathrm{degree}$ \\
$Q_{*}$ & mass ejection rate & $3.4 \times 10^{6}$ & $\mathrm{~kg} / \mathrm{hr}$ \\
$B$ & spacecraft mass to area ratio & $30^{*}$ & $\mathrm{~kg} / \mathrm{m}^{2}$
\end{tabular}

* assumed to be same as comet Wirtanen.

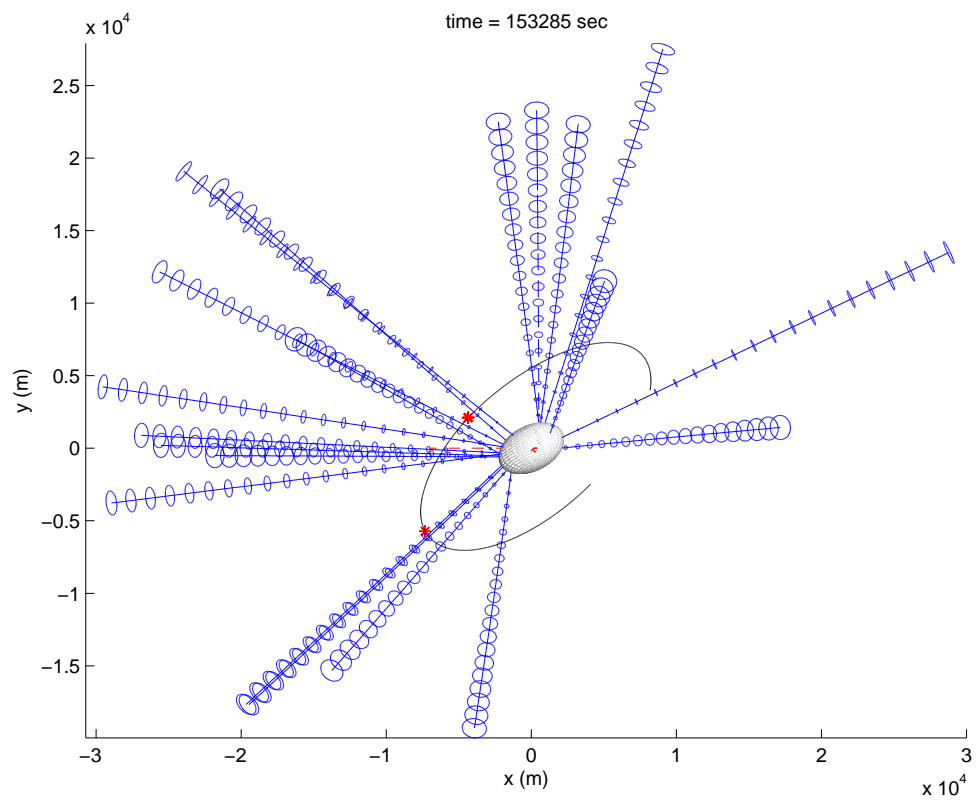

Figure 4. Spacecraft Trajectory in Inertial Frame for Interaction with Outgassing Jets with a Half Angle of $1.5^{\circ}$ and $V_{o g}$ of $0.5 \mathrm{~km} / \mathrm{s}$ on the Idealized $81 \mathrm{P} / \mathrm{Wild} 2$

The simulation is of the idealized comet $81 \mathrm{P} / \mathrm{Wild} 2$ (values in Table 1) where the mass ejection rate is from Neishtadt, et al, ${ }^{7}$ with 20 discrete outgassing jets located on the surface in the comet. Each jet is assumed to have a jet half angle of 1.5 degrees and an outgassing velocity of $0.5 \mathrm{~km} / \mathrm{s}$. This simulation is seen in Figs. 4 to 7 where the thicker sections of the spacecraft's trajectory are the locations of the jet passages. Figure 7 shows the changes in the orbital elements, semimajor axis and eccentricity, when the spacecraft encounters an outgassing jet. The two jet passages can clearly be seen by the jumps in the orbital elements. Since the spacecraft is located significantly close to the comet, we note that the radial impulse assumption may not hold for this simulation.

$$
9 \text { of } 16
$$




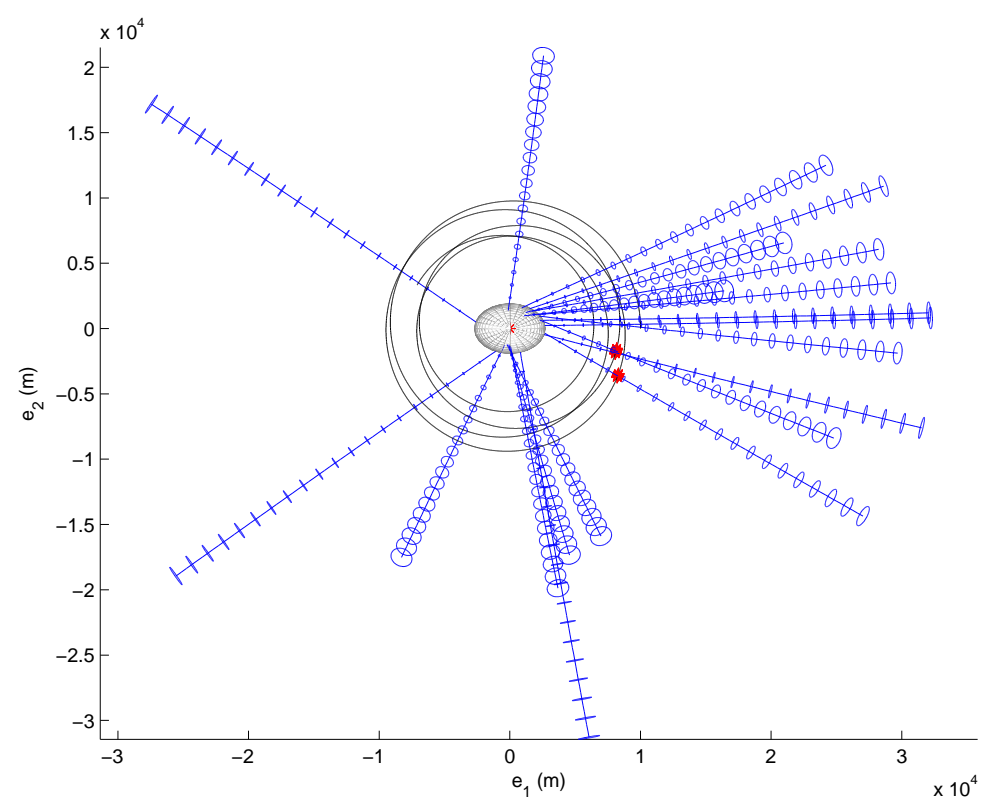

Figure 5. Spacecraft Trajectory in Body Fixed Frame for Interaction with Outgassing Jets with a Half Angle of $1.5^{\circ}$ and $V_{o g}$ of $0.5 \mathrm{~km} / \mathrm{s}$ on the Idealized $81 \mathrm{P} / \mathrm{Wild} 2$

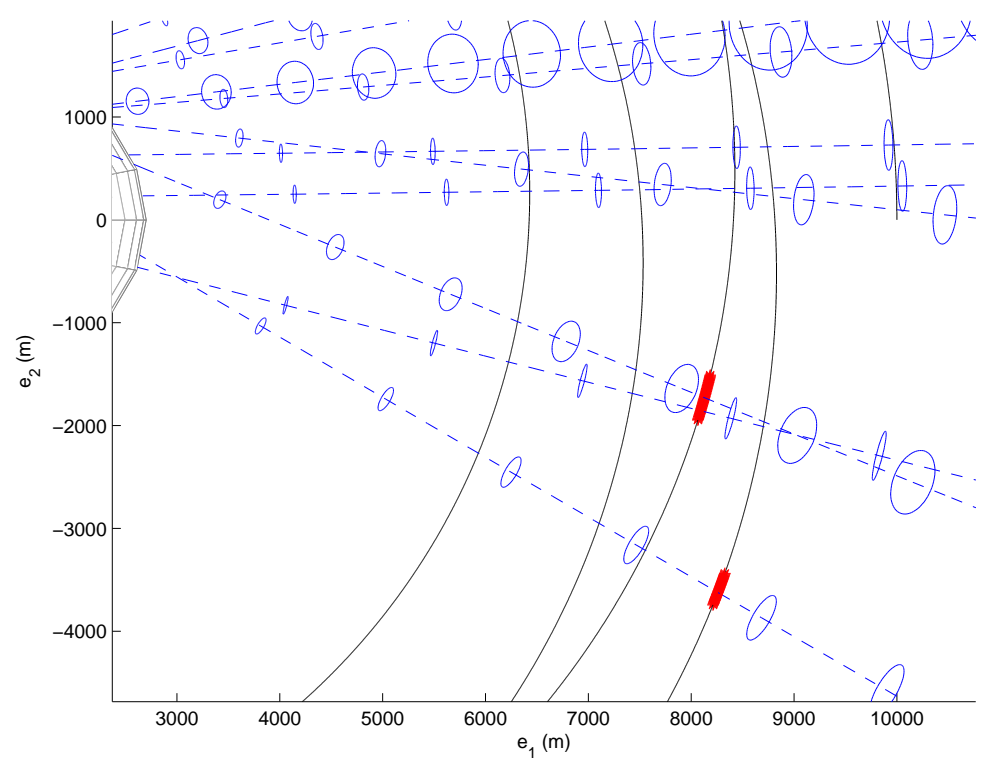

Figure 6. Spacecraft Trajectory in Body Fixed Frame for Interaction with Outgassing Jets with a Half Angle of $1.5^{\circ}$ and $V_{o g}$ of $0.5 \mathrm{~km} / \mathrm{s}$ on the Idealized $81 \mathrm{P} / \mathrm{Wild} 2$ (Detail)

\section{Estimation of Model}

The most important use of this model will be to aid in spacecraft navigation by providing a specific model with parameters that can be estimated from tracking data. This will both enable future trajectories to be predicted more accurately, and provide measurements of scientific interest. This model can be used to estimate the defining jet parameters for spacecraft passages through a discrete jet outgassing field. Based on Doppler tracking and a standard navigation solution for the spacecraft's trajectory, the jet's basic describing parameters, the jet location and the half angle, $\delta$, and the pressure of the outgassing, $|\vec{p}|$, can be estimated. 

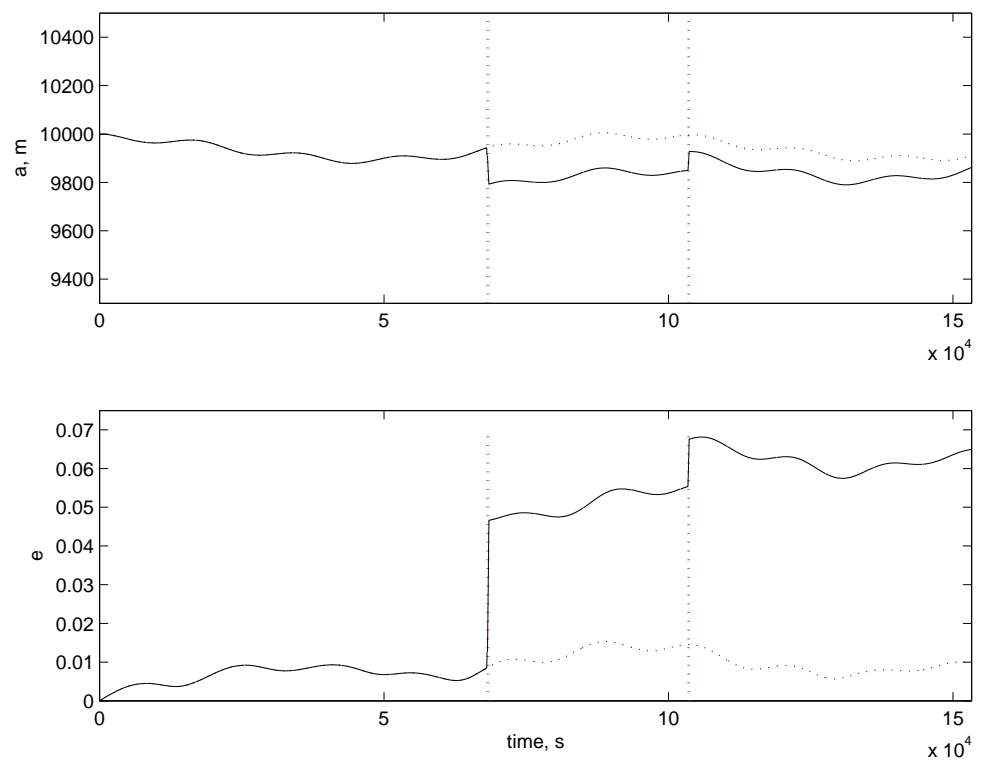

Figure 7. Semimajor Axis and Eccentricity for Spacecraft with Interaction with Outgassing Jets with a Half Angle of $1.5^{\circ}$ and $V_{o g}$ of $0.5 \mathrm{~km} / \mathrm{s}$ on the Idealized $81 \mathrm{P} /$ Wild2 with the Dotted lines Showing the Orbital Elements for Encountering No Jets. Variations are Due to the Non-Point Mass Gravity Field.

\section{A. Outgassing Acceleration on Spacecraft}

A spacecraft is assumed to begin passage through an outgassing field when the Doppler tracking detects an acceleration larger than $1.667 \times 10^{-5} \mathrm{~m} / \mathrm{s}^{2}$ (the accuracy to which Doppler data can unambiguously detect change in velocity). Since the acceleration is directly measured, if we can estimate $B$ and the mass flux rate, $Q_{j}$, (using a mass spectrometer) we can also gain a direct estimate of the outgassing speed using the relationship:

$$
\left|\overrightarrow{a_{p}}\right|=Q_{j} V_{o g} \frac{1}{B}\left(\frac{r_{0}}{\left|\vec{r}_{j}\right|}\right)^{2}
$$

In addition to estimating the outgassing velocity, this detection can also be used to identify boundary crossings of the jet outgassing field. These crossings can be used to yield unit vectors in the direction of a crossing in the body fixed frame, $\hat{u}_{i}$, to aid in estimation of other jet parameters assuming that a navigation solution for the spacecraft is available.

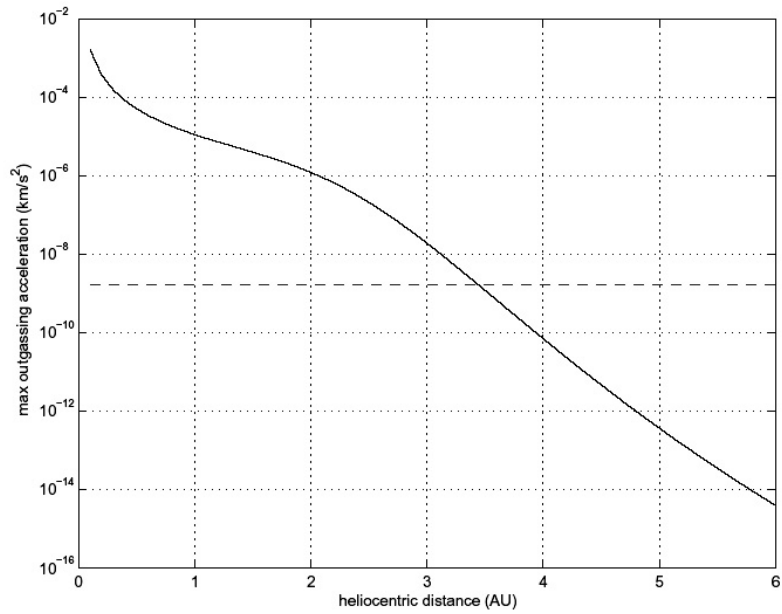

Figure 8. Outgassing Acceleration as a Function of Comet Orbital Radius (Doppler Tracking Threshold Shown as Dotted Line)

By assuming an outgassing velocity of $0.5 \mathrm{~km} / \mathrm{s}$ in the model, the magnitude of the outgassing acceleration can be detected by Doppler tracking when the comet's orbital radius is less then $3 \mathrm{AU}$ as seen in Fig. 8. The spikes in the spacecraft's acceleration components (seen in Fig. 9] from simulation data) illustrate jet passages. Since the magnitude of the acceleration spikes are on the order of $10^{-5} \mathrm{~m} / \mathrm{s}^{2}$, the jet passages should be able to be identified by the Doppler tracking.

\section{B. Jet Location and Half Angle, $\delta$}

With multiple jets emanating from the surface of the comet, it is necessary to appropriately identify each crossing with a possible jet location. These jet boundary crossings naturally occur in pairs, one crossing

$$
11 \text { of } 16
$$


classified as entering and one as exiting the outgassing field. These pairs can be mapped to the surface of the comet in the body fixed frame as unit vectors that lie on the surface boundary of the jet if the rotation of the comet and the outgassing velocity, $V_{o g}$, is assumed to be known. The time since ejection, $s$, can be found using the assumed outgassing velocity and the radial distance from the surface of the comet. This time gives the angular rotation of the comet from the rotation rate, $\omega_{\text {com }}$. Rotating the comet back in time and moving the crossing point to the surface of the comet gives the mapping method to a body fixed frame.

After the pairs have been mapped to the comet surface, they are identified with a possible jet location by checking the proximity of the unit vector which defines the pair's bisector with possible jets' estimated centerlines. If the bisector unit vector falls within the jet's estimated size (defined as the estimated half angle with some allowable tolerance) then the pair is grouped with other identified pairs for the possible jet location. If the bisector unit vector does not match with an existing possible jet location, the pair define a new possible jet location to be tested for subsequent crossings.

Once a crossing pair has been identified with a possible jet location, the number of pairs identified with that jet location determines the estimation process. If only a single pair has been identified, then the jet centerline location is assumed to be the unit vector which bisects the pair of unit vectors, and the half angle of the jet is estimated as half of the angle between the pair of crossing unit vectors.

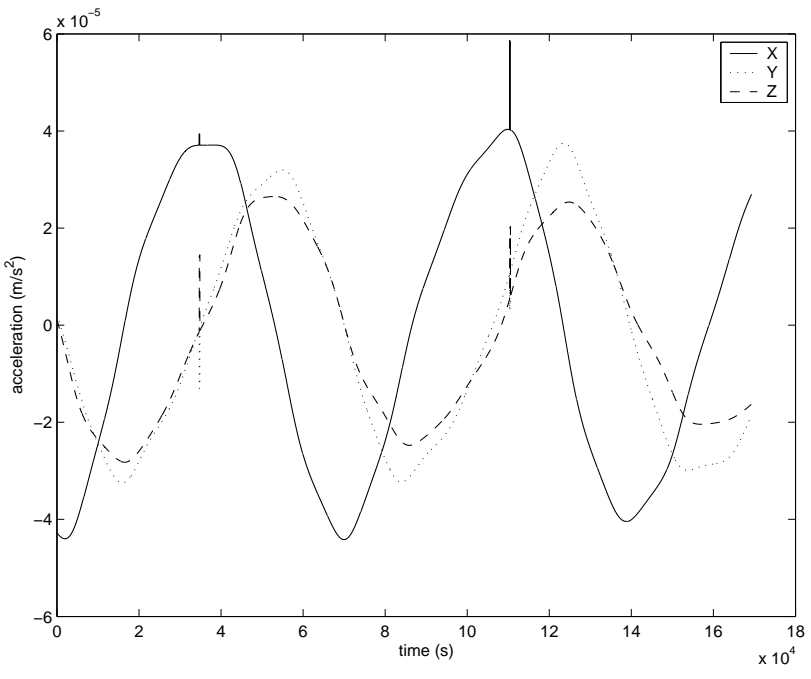

Figure 9. Spacecraft Acceleration Components in an Inertial Frame If multiple pairs are associated with a possible jet location, there are three possible methods to estimate the jet location and half angle, where two methods are based on the geometry of the circular cross section and the third utilizes the directly determined unit vectors. The first geometrical approach needs two pair of crossings to estimate the position and jet half angle. The pairs can be connected to form two chords. The crossing of the bisecting perpendiculars to these chords will give an estimate of the center of the jet. Using Pythagoras' theorem on one of the chords yields the estimate of the half angle as seen in Fig. 10. This estimate allows for the spacecraft to fly-through at different radii and allows for curved paths through the outgassing field. If the spacecraft is known to be in the plane of the outgassing jet then the chord constructed is the diameter of the jet cross section and reduces to the method used for a single pair crossing.

The second geometrical approach requires only one and a half crossing pairs of the jet outgassing field. Using the three unit vectors in the body fixed frame as vertices of a triangle, the geometrical formula for finding the radius of an circumscribed circle about a triangle can be used to estimate the jet half angle, $\delta$, as seen in Fig. 11 .

$$
\begin{gathered}
r_{0} \sin \delta=\frac{1}{2} \frac{a_{t} b_{t} c_{t}}{\sqrt{s\left(s-a_{t}\right)\left(s-b_{t}\right)\left(s-c_{t}\right)}} \\
s=\frac{1}{2}\left(a_{t}+b_{t}+c_{t}\right)
\end{gathered}
$$

where $a_{t}, b_{t}$, and $c_{t}$ are the chord lengths created by connecting the three crossings. Once the half angle is known, the center of the jet can be inferred by finding the common intersection point of circle of radius $\delta$ drawn about each crossing point. This approach could be used if one of the boundary crossings is ill-defined.

The third approach is the most general and allows for non-circular cross-sections of the outgassing jet to be ideally estimated. Using the mapped unit vectors in the body fixed frame in the pairs of crossings (illustrated in Fig. 12), another unit vector pointing in the direction of the estimated center of the cross- 
section can be defined. The nominal cone angle is found as:

$$
\hat{u}_{i} \cdot \hat{u}_{c}=\cos (\delta)
$$

where $\hat{u}_{i}$ is a unit vector in the direction of a crossing and $\hat{u}_{c}$ is a unit vector in the direction of the crosssection's center. A pair of boundary crossings will produce a plane of possible solutions to $\hat{u}_{c}$. The position of the center of the outgassing jet can be estimated by a third crossing which gives a unique solution to $\hat{u}_{c}$ and thus the half angle, $\delta$.

This estimate for a $\hat{u}_{c}$, and hence $\delta$, can be refined when subsequent boundary crossings occur by using a least squares approach, where the cost function is defined as:

$$
J=\frac{1}{2} \sum_{i=1}^{N}\left(\hat{u}_{i} \cdot \hat{u}_{c}-\cos \delta\right)^{2}
$$

Taking the partial derivative with respect to the center unit vector and setting it equal to zero yields an estimate for $\hat{u}_{c}$.

$$
\begin{aligned}
\frac{\partial J}{\partial \hat{u}_{c}} & =\sum_{i=1}^{N}\left(\hat{u}_{i} \cdot \hat{u}_{c}-\cos \delta\right) \hat{u}_{i}=0 \\
\vec{u}_{c} & =\cos \delta\left(\sum_{i=1}^{N} \hat{u}_{i} \hat{u}_{i}\right)^{-1} \sum \hat{u}_{i}
\end{aligned}
$$

This solution should point in the direction of the proper solution, but may not be a unit vector. Normalizing the estimate for $\overrightarrow{u_{c}}$ yields a function only of the boundary crossing unit vectors.

$$
\hat{u}_{c}=\frac{\left(\sum_{i=1}^{N} \hat{u}_{i} \hat{u}_{i}\right)^{-1} \sum \hat{u}_{i}}{\left|\left(\sum_{i=1}^{N} \hat{u}_{i} \hat{u}_{i}\right)^{-1} \sum \hat{u}_{i}\right|}
$$

Note that for $N<3$, the term $\sum_{i=1}^{N} \hat{u}_{i} \hat{u}_{i}$ will not be invertible in general, but that for $N \geq 3$ for a properly chosen set of $\hat{u}_{i}$ will produce a unique solution. Taking the partial derivative of the cost function with respect to the jet half angle, $\delta$, yields an estimate for the half angle which is dependent on the estimate for the center unit vector found above in Eq. 61 .

$$
\begin{gathered}
\frac{\partial J}{\partial \delta}=\sum_{i=1}^{N}\left(\hat{u}_{i} \cdot \hat{u}_{c}-\cos \delta\right) \sin \delta \\
\cos \delta=\frac{1}{N}\left(\sum_{i=1}^{N} \hat{u}_{i}\right) \cdot \hat{u}_{c} \\
\delta=\arccos \left(\frac{1}{N} \hat{u}_{c} \cdot \sum_{i=1}^{N} \hat{u}_{i}\right)
\end{gathered}
$$

These new estimates for the jet centerline unit vector and half angle are used to identify subsequent jet crossing pairs with the jet. This iterative process

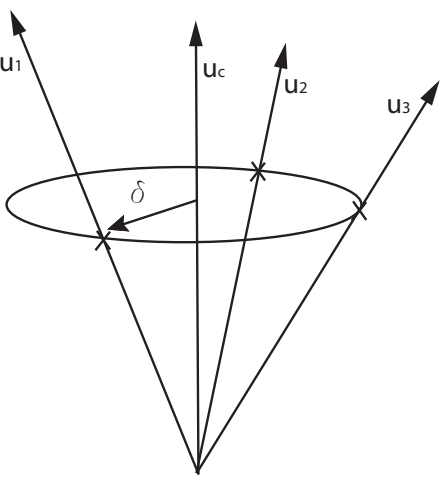

Figure 12. Body Fixed Unit Vectors Method of Estimating Jet Half Angles allows for the mapped pairs to be identified properly to the correct jet.

Once the location and half angle of the jets have been estimated, the mapped boundary crossing vectors can be intersected with the surface of the comet to define the boundary of an active surface patch associated with the jet. Correlating this surface patch with images of the comet's surface can yield insight into the orientation or source of the jet from surface features such cavities or sharp inclines. The errors in these estimations can be caused by uncertainty in the spacecraft's position, uncertainty in the comet's rotation, or from lack of detailed knowledge of the outgassing jet's structure. Uncertainty in the spacecraft's position and in the comet's rotation can produce errors in the mapped unit vectors yielding errors in the boundary crossings. Lack of knowledge of the jet's structure can lead to errors in the identification of jet locations. One such scenario is the jet's outgassing pressure profile not being a solid cone of pressure but is instead is a hollow cone producing more "boundary crossings" then actually exist, yielding the appearance of crossing two jets instead of one. 


\section{Estimation Simulation}

Utilizing the model simulation, the boundary crossing positions and times are collected and passed on to estimate the jet parameters. For the estimation of the jet half angle, $\delta$, the estimation simulation utilizes the unit vector least squares approach to estimate the jet center unit vector and the jet half angle assuming that the outgassing velocity and comet rotation is already known. We note that the locations of possible jets are not assumed and are estimated as well. If only a single pair has been identified with a possible jet location, then the half angle of the jet is estimated as half of the angle between the pair of boundary crossing unit vectors. Using simulation data for a half angle of 1.5 degrees and outgassing velocity of $0.5 \mathrm{~km} / \mathrm{s}$ for multiple jets, seen in Fig. 13, the jet half angle is estimated by changing the maximum allowable angle error in the simulation of the spacecraft's trajectory. The maximum allowable angle error is defined as the largest angle with respect

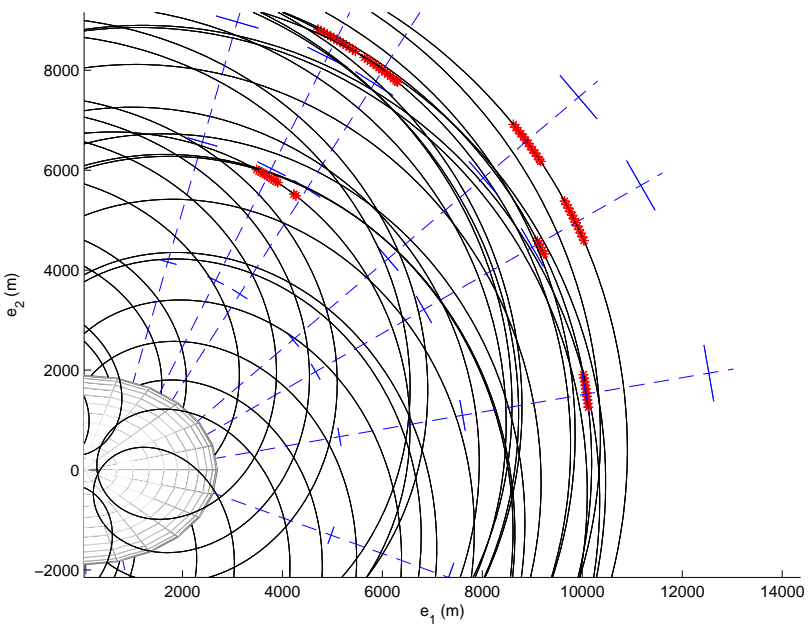

Figure 13. Simulation of Jets with Half Angle of 1.5 degrees and Outgassing Velocity of $0.5 \mathrm{~km} / \mathrm{s}$ (detail) to the jet boundary such that the spacecraft is considered to have crossed the boundary and replicates the spacecraft's measurement error. For the half angle

Table 2. Outgassing Half Angle Estimation (degrees)

\begin{tabular}{ccccc} 
Jet & Crossings & \multicolumn{3}{c}{ Maximum Angle Error } \\
No. & pts. & 1 degree & 0.5 degree & 0.1 degree \\
\hline 1 & 2 & 2.3337 & 2.3337 & 2.5458 \\
2 & 4 & 2.3120 & 2.4327 & 2.8134 \\
3 & 4 & $0.6354^{*}$ & 2.2614 & 2.4815 \\
4 & 2 & 1.9073 & 1.9073 & 1.9073 \\
5 & 2 & 0.4227 & 0.4227 & 0.4227 \\
6 & 2 & 1.6908 & 1.6908 & 1.6908 \\
7 & 2 & 1.9236 & 1.9236 & 2.1373 \\
8 & 4 & 2.9108 & 2.9108 & 1.9160 \\
9 & 2 & 2.4646 & 2.4646 & 2.2406 \\
10 & 2 & 0.4206 & 0.6309 & 0.8412 \\
11 & 2 & 2.5279 & 2.5279 & 2.5279 \\
12 & 2 & 1.6857 & 1.4750 & 1.6857 \\
$*$ & estimated with two crossing points. &
\end{tabular}

estimate, a small angle error in the measurement results, in general, in a smaller half angle estimate error as seen in Table 2. The exceptions are caused by the time step used in the simulated data. The errors in the estimation can be caused by uncertainty in the spacecraft's position, uncertainty in the comet's rotation, or from lack of detailed knowledge of the outgassing jet's structure discussed previously.

\section{Estimation Applications}

The described estimation methods have many applications as navigation tools for a spacecraft. The estimates can be used to identify jets and map their locations on the surface of the comet as well as determine their size. These parameters can give the spacecraft better trajectory fits and predictions. The location of the jet also allows for targeted scientific measurements, turning on measurement instruments only when the spacecraft is in or near a jet outgassing field. In conjunction with mass spectrometer measurements, we can determine the 
gas velocity with simple relationships. This also holds for a given gas velocity from which we can determine the gas mass flux. Applications of the outgassing field and jet structure can also be obtained once the field boundary has been crossed. The spacecraft can measure the variation of the outgassing within a jet's field. It may not be a solid cone structure as assumed in the model, but may actually have an inactive or less active interior. Multiple passes can uncover complex jet boundaries that may be related to the outgassing conditions at the comet surface.

\section{Conclusions}

This paper has presented a simple navigation model for a single discrete jet with constant half angle, $\delta$, using a uniform spherical model of the comet. The model of the jet was simulated using 20 discrete jets to verify the results found in the changes in the orbital elements. From the small changes in the orbital parameter and the orbital energy, it was shown that if the spacecraft has a negative radial velocity component, the outgassing jet will decrease the semimajor axis and eccentricity and if the spacecraft has a positive radial velocity component, the outgassing jet will tend to make the orbit more eccentric and increase the semimajor axis for a radial impulse when the spacecraft is located significantly far from the comet.

Estimation methods were presented for the outgassing pressure accelerations, the half angles, and the locations of jets. The outgassing pressure can be determined by taking a measurement of the mass flux and assuming an the outgassing velocity. The half angle can be estimated by using boundary crossing points mapped to the surface of the comet. The method for estimating the half angle of the jet includes a identifying jets when multiple jet outgassing fields have been crossed. For the half angle estimation method simulated, a small angle error in the measurement results in general in a small half angle estimate error.

\section{Appendix}

The jet locations and orientations in Table A-1 are from Sekanina, et al. ${ }^{4}$

\section{Acknowledgments}

This research was funded, in part, by a grant from the Jet Propulsion Laboratory/California Institute of Technology Director's Research and Development Fund.

\section{References}

\footnotetext{
${ }^{1}$ Miller, J. K., Weeks, C. J., and Wood, L. J., "Orbit Determination Strategy and Accuracy for a Comet Rendezvous Mission," Journal of Guidance, Control, and Dynamics, Vol. 13, No. 5, 1990, pp. 775-784.

${ }^{2}$ Scheeres, D. J., Marzari, F., Tomasella, L., and Vanzani, V., "ROSETTA mission: satellite orbits around a cometary nucleus," Planet. Space Sci., Vol. 46, No. 6/7, 1998, pp. 649-671.

${ }^{3}$ Crifo, J. F., Itkin, A. L., and Rodionov, A. V., "The Near-Nucleus Coma Formed by Interacting Dusty Gas Jets Effusing from a Cometary Nucleus: I," ICARUS, Vol. 116, 1995, pp. 77-112.

${ }^{4}$ Sekanina, Z., Brownlee, D. E., Economou, T. E., Tuzzolina, A. J., and Green, S. F., "Modeling the Nucleus and Jets of Comet 81P/Wild 2 Based on the Stardust Encounter Data," Science, Vol. 304, No. 5678, 2004, pp. 1769-1774.

${ }^{5}$ Scheeres, D. J., "Dynamics About Uniformly Rotating Tri-Axial Ellipsoids. Applications to Asteroids," ICARUS, Vol. 110, 1994, pp. 225-238.

${ }^{6}$ Combi, M. R., Kabin, K., DeZeeuw, D. L., and Gombosi, T. I., "Dust-Gas Interrelations in Comets: Observations and Theory," Earth, Moon, and Planets, Vol. 79, 1997, pp. 275-306.

${ }^{7}$ Neishtadt, A. I., Scheeres, D. J., Sidorenko, V. V., and Vasiliev, A. A., "Evolution of Comet Nucleus Rotation," ICARUS, Vol. 157, 2002, pp. 205-218.

${ }^{8}$ Marsden, B. G., Sekanina, Z., and Yeomans, D. K., "Comets and nongravitational forces," Astronomical Journal, Vol. 78, 173, pp. 211-225.

${ }^{9}$ Klinger, J., Levasseur-Regourd, A., Bouziani, N., and Enzian, A., "Towards a model of cometary nuclei for engineering studies for future space missions to comets," Planet. Space Sci., Vol. 44, No. 7, 1996, pp. 637-653.
} 
Table A-1. Jet Locations and Orientations

Orientation Angles Jet Location Angles degrees degrees

\begin{tabular}{ccccc} 
Jet & $\psi_{\text {or }}$ & $\lambda_{\text {or }}$ & $\phi_{\text {loc }}$ & $\lambda_{\text {loc }}$ \\
\hline$\alpha$ & 161 & 4 & 169 & 1 \\
$\beta$ & -7 & 36 & 16 & 14 \\
$\gamma$ & 25 & 26 & 38 & 13 \\
$\delta$ & 22 & 59 & 42 & 38 \\
$\epsilon$ & -15 & -20 & 353 & -7 \\
$\zeta$ & 9 & 23 & 23 & 9 \\
$\eta$ & -65 & 3 & 294 & 2 \\
$\theta$ & -130 & 4 & 238 & 2 \\
$\kappa$ & 40 & 31 & 60 & 19 \\
$\lambda$ & 30 & 47 & 45 & 28 \\
$\mu$ & -59 & 56 & 266 & 43 \\
$\nu$ & -49 & 57 & 260 & 43 \\
$\xi$ & 20 & 25 & 27 & 10 \\
$\pi$ & 34 & 17 & 45 & 9 \\
$\rho$ & -64 & 51 & 272 & 38 \\
$\sigma$ & 97 & 49 & 95 & 36 \\
$\tau$ & -101 & 53 & 252 & 39 \\
$\phi$ & 16 & -10 & 14 & -3 \\
$\chi$ & 16 & -10 & 5 & -3 \\
$\psi$ & 1 & -7 & 350 & -2
\end{tabular}

16 of 16 